\title{
VARIABLES PREDICTORAS DE LA EXPECTATIVA DE DESEMPEÑO Y LA INTENCIÓN DE ABANDONO EN CONTEXTO DE EDUCACIÓN VIRTUAL DE EMERGENCIA EN ESTUDIANTES UNIVERSITARIOS CHILENOS
}

\author{
Predictive Variables of Performance Expectation and Intention to Drop Out in \\ the Context of Virtual Emergency Education in Chilean College Students
}

Jorge Maluenda-Albornoz ${ }^{1}$

jorgemaluendaa@gmail.com

\section{Ana B. Bernardo²}

bernardoana@uniovi.es

Celia Galve-González²

celiagalvegon@gmail.com

\section{Gabriela Flores-Oyarzo ${ }^{1}$}

ps.gabrielafloresoyarzo@gmail.com

Valeria Infante-Villagrán ${ }^{1}$

valeria.a.infante.v@gmail.com

Alejandro Díaz-Mujica ${ }^{1}$

adiazm@udec.cl

\footnotetext{
${ }^{1}$ Universidad de Concepción. Chile

${ }^{2}$ Universidad de Oviedo. España
}

Recibido: 4/03/2021

Revisado: 4/05/2021

Aprobado: 11/05/2021

Preprint: 8/06/2021

Publicado: 15/07/2021

\section{Resumen}

El actual contexto de educación virtual de emergencia producto de la pandemia del COVID19 ha traído nuevas condiciones al escenario educativo. En este contexto, esta investigación busca evaluar la capacidad predictiva de las expectativas de compromiso, el apoyo social, la calidad y dominio de los recursos informáticos, las Notas de Enseńanza Media (NEM) y el puntaje obtenido en la Prueba de Selección Universitaria (PSU) sobre las expectativas de desempeño y la intención de abandono durante el primer semestre académico del año 2020, en estudiantes universitarios chilenos. Se realizó un diseńo asociativo de tipo predictivo con una medición transversal. Participaron 347 estudiantes. Los resultados mostraron que el dominio y la calidad percibida de los recursos informáticos, el apoyo social percibido, las NEM y la PSU no son predictores significativos de las expectativas de desempeño y la intención de abandono, a diferencia de las expectativas de compromiso y de desempeño. La PSU, el apoyo social y las expectativas de compromiso fueron predictoras de las expectativas de desempeño académico.

Palabras clave: abandono de estudios, educación a distancia, estudiante universitario, expectativa de desempeño, desempeño académico.

\section{Abstract}

The current context of virtual emergency education due to the COVID19 pandemic has brought new conditions to the educational scene. In this context, this research sought to evaluate the predictive capacity of engagement expectations, social support, the quality and mastery of computer resources, the High School Grades (NEM) and the score obtained in the University Selection Test (PSU) on performance expectations and the intention to drop out during the first academic semester of 2020, in Chilean university students. A predictive associative design was carried out with a cross-sectional measurement. 347 students participated. The results showed that the mastery and the perceived quality of computer resources, the perceived are not significant predictors of performance expectations and intention to drop out, as opposed to engagement and performance expectations. The PSU, social support and engagement expectations were shown to predict academic performance expectations.

Keywords: academic performance, achievement, distance education, dropout, performance expectation, university student.

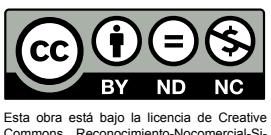

Esta obra está bajo la licencia de Creative nObraDerivada 4.0 Internacional.
nomitis

ISSN (impreso): 2636-2139

ISSN (en línea): 2636-2147

Sitio web: https://revistas.isfodosu.edu.do/recie 


\section{Introducción}

El actual contexto de educación virtual de emergencia producto de la pandemia del COVID19 ha traído nuevas condiciones al escenario educativo. Los problemas vinculados al desempeño y el abandono de los estudiantes pueden generar condiciones especiales en este nuevo escenario, dada la importancia del acceso y manejo del internet y los recursos informáticos.

Diversas variables se vinculan con la intención de abandono, algunas relacionadas al perfil de ingreso, como el puntaje en la Prueba de Selección Universitaria (PSU) y las Notas de la Enseñanza Media (NEM), y otras propias de la experiencia socioacadémica, como el compromiso académico, el apoyo social y la calidad y dominio de los recursos informáticos.

La presente investigación busca evaluar la capacidad predictiva de las expectativas de compromiso, el apoyo social, la calidad y dominio de los recursos informáticos, las NEM y la PSU sobre las expectativas de desempeńo y la intención de abandono durante este semestre académico, en estudiantes universitarios chilenos.

Se entiende por intención de abandono aquella disposición que manifiesta un estudiante a desvincularse de la universidad (Duque et al., 2014) antes de la consecución de su titulación, y por expectativa de desempeńo, aquella idea o imaginario que tiene el estudiante respecto de cómo será su rendimiento académico en el contexto y circunstancias que enfrenta actualmente, teniendo en consideración sus experiencias previas y sus características y recursos personales.

Para medir la capacidad predictiva de las variables previamente mencionadas sobre la intención de abandono y la expectativa de desempeño, se cuenta con la participación voluntaria de 374 estudiantes universitarios chilenos de la región del Biobío, quienes respondieron a los ítems de un instrumento construido ad hoc por el equipo investigador. El nivel de compromiso académico se midió en 3 ítems, así como también la expectativa de desempeño, la intención de abandono, el apoyo social percibido, la calidad de las herramientas informáticas y el nivel de dominio de los recursos tecnológicos. Por su parte, las NEM y el puntaje obtenido en la PSU se solicitaron por medio del autorreporte.

\section{Revisión de la literatura}

Las especiales condiciones en que se desarrolla actualmente la educación universitaria producto de la pandemia del COVID19 han forzado a una modalidad de educación caracterizada por una adaptación permanente de las condiciones de acceso y dominio tecnológico en el desarrollo del proceso educativo, así como de acomodamiento de los métodos educativos utilizados, en los que la disposición, la calidad y el dominio de aparatos electrónicos con conectividad a Internet se vuelven esenciales (UNESCO, 2020). Estas condiciones han provocado que estudiantes con distinto perfil socioeconómico y académico se enfrenten al estudio en condiciones de confinamiento permanente y de aislamiento social.

En un contexto de educación virtual, el escaso dominio y acceso tecnológico pueden ser factores que impulsen a los estudiantes a abandonar los estudios (Orozco et al., 2017). En esa misma línea, el bajo desarrollo de competencias digitales parece impactar indirectamente en el desempeño académico (Moneta, 2019), haciendo que la educación a distancia requiera un mayor compromiso por parte del estudiantado (UNESCO, 2020).

En condiciones "regulares" se ha observado que las variables asociadas al perfil de ingreso del estudiante están vinculadas a la intención de abandono de los estudios, entre ellas el puntaje obtenido en la PSU (Jorquera et al., 2018) y las NEM (Vergara et al., 2016) han sido predictores significativos del abandono y han estado asociados al desempeño del estudiante (Tuero et al., 2018).

La experiencia socioacadémica, por su parte, también ha sido de gran relevancia frente al desempeño y el abandono. El compromiso académico, entendido como la disposición del estudiante a esforzarse en dominar habilidades y contenidos complejos, a 
participar activamente en actividades académicas y a reaccionar positivamente frente a sus pares y docentes (Maroco et al., 2016), ha demostrado ser un predictor significativo de la intención de abandono (Díaz-Mujica et al., 2018; López-Angulo et al., 2019; Maluenda et al., 2020). Implica la disposición de diversos recursos por parte del estudiante mientras cursa estudios, entre los cuales se encuentran los siguientes: energía y resistencia mental; dedicación, expresada en entusiasmo e inspiración al estudiar; y concentración total en el trabajo, la cual se experimenta con agrado (Díaz-Mujica et al., 2019).

Otro factor relevante es el apoyo social percibido tanto por parte de los pares como de los docentes, el cual puede entenderse como aquella percepción que tiene el estudiante respecto de la calidad y cantidad de apoyo social disponible (Cohen \& Janicki-Deverts, 2010; Orcasita \& Uribe, 2010), y va a favorecer su integración tanto al contexto social como al académico, influyendo no solo el compromiso (Maluenda et al., 2020), sino también, de manera indirecta, la intención de abandono.

Por otro lado, al ingresar al contexto universitario el estudiante lo hace con una serie de características, metas, expectativas y compromisos personales que impactarán en su experiencia en educación superior, las cuales al interactuar con las experiencias de integración social y académica se verán modificadas para dar paso a la construcción de nuevos objetivos, metas y compromisos en torno a su educación y a la institución en que cursa sus estudios (Ayala \& Atencio, 2018; Benítez-Zavala, 2016; England, 2012; Saldaña \& Barriga, 2010; Schmitt \& Santos, 2016). De manera que las metas que tiene cada estudiante van a dirigir el esfuerzo que realiza para obtener la titulación, y de esa manera guardan relación con la adaptación académica (Díaz-Mujica et al., 2019) y con la intención de abandono (Maluenda et al., 2019).

De manera que el perfil de ingreso, el compromiso académico, el apoyo social percibido y las expectativas y metas de los estudiantes son variables que impactarían de manera directa o indirecta en la intención de abandono y las expectativas de desempeño académico.

La intención de abandono se entiende como el conjunto de pensamientos que tiene un estudiante, en el transcurso de su carrera y antes de alcanzar la titulación, respecto a cesar su permanencia en el programa formativo que cursa actualmente y que le conduce a obtener un título en Educación Superior (Díaz-Mujica et al., 2018).

La expectativa de desempeño académico, por su parte, se comprende a partir de la integración de la conceptualización de desempeño académico y expectativa. El desempeño académico se entiende como el conjunto de indicadores que dan cuenta de la efectividad y grado en que se han logrado los objetivos propuestos en los programas de estudio constituyéndose como todo comportamiento realizado por el estudiante a fin de cumplir con las responsabilidades asignadas o autoasignadas (Ortega-Medellín, 2020). Las expectativas se comprenden como todo aquello que los estudiantes esperan realizar y concretar durante su vida académica, se relacionan con todo aquello que, según sus experiencias previas, les sugiere que es más probable que ocurra, y conforman una interpretación y predicción de lo que sucederá, con lo que transforman conocimientos, actitudes, motivaciones y creencias, en acciones y comportamientos (Deaño et al., 2014).

De manera que la expectativa de desempeño se entiende como aquel imaginario que tiene el estudiante respecto de lo que es y será su desempeño académico, fundamentado en sus experiencias educativas previas, y del autoanálisis y evaluación de sus cualidades, fortalezas, debilidades y recursos (humanos y materiales) a la hora de enfrentar tareas académicas.

Cabe señalar que las expectativas juegan un rol clave en la predicción de la decisión de continuar o abandonar los estudios. Sin embargo, no son estáticas, sino que son modificables y se pueden ajustar. Conocerlas permite identificar tempranamente las necesidades individuales de los estudiantes, personalizar intervenciones y promover el éxito (Deaño et al., 2014). 
Dado que la relevancia del estudio de estas variables no radica solo en su capacidad predictiva de fenómenos como el abandono, sino también en su susceptibilidad de modificación, lo cual implica que son un recurso para el diseño de intervenciones que permitan mejorar la permanencia estudiantil (Maluenda et al., 2019), y considerando que las expectativas que tienen los estudiantes cumplen un rol importante en la predicción de su conducta (Deańo et al., 2015; Pleitz et al., 2015), sumado a las características del actual contexto educativo, es relevante indagar si las variables señaladas influyen del mismo modo que en la educación regular sobre la intención de abandono y las expectativas de desempeño académico. También es relevante conocer el rol que juegan las variables propias de la virtualidad, como la calidad y el nivel de dominio de las herramientas tecnológicas por parte de los estudiantes.

El objetivo de la presente investigación fue evaluar la capacidad predictiva de las expectativas de compromiso, el apoyo social, la calidad y dominio de los recursos informáticos, las NEM y la PSU, sobre las expectativas de desempeño y la intención de abandono en estudiantes universitarios chilenos en el contexto de la educación de emergencia.

\section{Método}

\subsection{Participantes}

Se realizó un muestreo intencionado enviando la invitación a participar mediante correo electrónico a todos los estudiantes de la universidad participante, en abril del año 2020. Esto fue durante el primer mes de clases en el período de pandemia del COVID 19. Accedieron a participar 374 estudiantes, 43,6\% hombres, 55,9\% mujeres, $0,5 \%$ que se identificaron en otra categoría, con edades de entre 17 y 25 años $(\mathrm{X}=19, \mathrm{SD}=2,3)$.

\subsection{Diseńo y procedimientos}

Se realizó una investigación con diseño asociativo de tipo predictivo (Ato et al., 2013). Se solicitó la participación voluntaria mediante el envío de un cuestionario digital en Google Forms a estudiantes de una universidad compleja perteneciente al Consejo de Rectores de Chile, ubicada en la región del Biobío.

Los estudiantes respondieron este instrumento durante el mes de abril del año 2020, luego de la confirmación digital de un consentimiento informado en el cual se explicaron los objetivos y características de la investigación.

Los resultados fueron analizados a partir de la exploración preliminar de las correlaciones entre las variables, para luego realizar la evaluación de un modelo de regresión lineal posterior al testeo y cumplimiento de los supuestos asociados a estas pruebas de estadística inferencial. El software utilizado para la realización de los análisis fue el SPSS v.21.

El análisis de los datos se realizó a partir de la observación de los estadísticos descriptivos y el método de regresión lineal múltiple (RLM). Se realizó la RLM teniendo como variables independientes las expectativas de compromiso, apoyo social, calidad y dominio de recursos informáticos, NEM y PSU, y como variable dependiente las expectativas de desempeño, para averiguar si las variables son predictores significativos de las expectativas de desempeño durante el primer semestre de la educación virtual de emergencia.

Se realizó RLM teniendo como variables independientes el apoyo social, la calidad y dominio de recursos informáticos, NEM y PSU, y como variable dependiente las expectativas de compromiso, con la finalidad de evaluar si estas variables predicen la expectativa de compromiso durante el primer semestre de la educación virtual de emergencia.

\subsection{Instrumentos}

La medición se realizó utilizando ítems construidos ad-hoc por los investigadores para evaluar las respuestas de los estudiantes sobre el curso de las actividades académicas durante el primer semestre del año 2020, en condiciones de emergencia sanitaria y educación virtual de emergencia.

Las distintas variables del presente estudio se evaluaron a partir de 3 preguntas para cada variable, con un 
formato de respuesta en escala Likert de 7 alternativas $(1=$ valor mínimo; 7 = valor máximo $)$.

Se evaluó la forma en que perciben su nivel de compromiso académico durante el semestre a partir de 3 ítems. Los ítems utilizados se basaron en el University Student Engagement Inventory, USEI (Maroco et al., 2016), que ha sido validado en la población de estudio (Maluenda et al., 2020). Los ítems utilizados fueron: a) "Espero que mi nivel de esfuerzo en las actividades académicas durante este semestre sea", b) "Espero que mi grado de participación en las actividades académicas durante este semestre sea", c) "Espero que mi nivel de interés en las actividades académicas durante este semestre sea". La escala de respuesta para esta pregunta fue la siguiente: $1=$ Muy bajo; 7 = Muy alto.

La expectativa de desempeńo también consideró 3 ítems orientados a evaluar el nivel de desempeño que tendrían durante el primer semestre académico. Los ítems considerados fueron los siguientes: a) "Creo que mi desempeño académico este semestre será, b) "Creo que mis calificaciones durante este semestre serán", c) "Creo que los resultados que obtendré este semestre serán”. La escala de respuesta fue la siguiente: 1 = Muy baja/s; 7 = Muy alta/s).

El apoyo social percibido se evaluó a través de 3 ítems: un ítem para considerar el apoyo social percibido de los compańeros, otro para el de los profesores y otro para el de su red familiar. Los ítems utilizados fueron los siguientes: a) "Creo que cuento con algún/ os compañero/s de la carrera/titulación que me apoyarían en caso de necesitarlo", b) "Creo que cuento con algún/os docente/s que me apoyaría/n en caso de necesitarlo", c) "Cuento con algún/os familiar/es que me ayudarían en caso de necesitarlo". El formato de respuesta fue la siguiente: 1 = Muy en desacuerdo; 7 $=$ Muy de acuerdo.

La calidad percibida de las herramientas informáticas se pesquisó a partir de 3 ítems que consideran el acceso a computador e internet de una calidad suficiente para realizar las labores estudiantiles. Los ítems utilizados fueron los siguientes: a) "Creo que la calidad del computador que uso para realizar las labores estudiantiles es", b) "Creo que la calidad del internet con que cuento para realizar mis labores académicas es", c) "Creo que la calidad de los recursos tecnológicos con los que cuento para realizar apropiadamente mis labores académicas es". La forma de respuesta para las preguntas fue la siguiente: $1=$ Muy baja; 7 = Muy alta.

El nivel de dominio de los recursos tecnológicos se evaluó también a partir de 3 ítems sobre el nivel de habilidad percibida por el estudiante respecto del uso de los recursos que requiere para poder cursar sus estudios. Las preguntas utilizadas fueron: a) "Mi nivel de dominio de las herramientas tecnológicas que estoy necesitando para estudiar y mantener mi actividad académica actual es", b) "Mi nivel de conocimiento sobre las herramientas tecnológicas básicas para estudiar en un nivel es", c) "Creo que mi habilidad para trabajar con las herramientas tecnológicas necesarias para estudiar actualmente es". La forma de respuesta para las preguntas fue la siguiente: $1=$ Muy bajo; 7 = Muy alto.

Las calificaciones de la enseńanza media (NEM) son las calificaciones oficiales que los estudiantes obtuvieron durante su escolaridad media/secundaria. Se solicitó a los estudiantes indicar cuál fue el promedio oficial que obtuvieron. Los estudiantes debieron indicar el promedio de calificaciones obtenido durante este período de estudio, el cual se registra en una escala de 1 a 7 acorde al sistema nacional de calificaciones chileno. Asimismo, el puntaje de la Prueba de Selección Universitaria es aquel que los estudiantes obtienen en la prueba nacional realizada para el ingreso a la universidad en Chile. Se solicitó a los estudiantes indicar el puntaje oficial obtenido en esta prueba. Este puntaje tiene un mínimo de 250 puntos y un máximo de 850 puntos, acorde al sistema nacional de calificaciones chileno.

Por último, se incluyeron 3 ítems para la intención de abandono también validados en la población de estudio (Díaz-Mujica et al., 2018), que miden la intención que tiene un estudiante de abandonar la universidad en la que se encuentra cursando sus estudios. Los ítems incluidos fueron: a) "Estoy pensando 
en abandonar esta universidad", b) "No deseo continuar mis estudios en esta universidad", c) "Quiero finalizar mis estudios en esta universidad". El formato de respuesta fue el siguiente: 1 = Muy en desacuerdo; 7 = Muy de acuerdo.

\section{Resultados}

A partir de los datos obtenidos se evaluaron los supuestos asociados a la regresión lineal múltiple y se cumplieron los criterios establecidos por la literatura para su realización. Luego se evaluaron dos modelos: un modelo para evaluar el valor predictivo de las distintas variables consideradas sobre la expectativa de desempeño durante el primer semestre académico, y otro para evaluar su valor predictivo sobre la intención de abandono de los estudios universitarios durante el mismo semestre.

El testeo de un modelo de regresión lineal múltiple para predecir la expectativa de desempeño arrojó un porcentaje de la varianza explicada en esta variable del $30 \%(\mathrm{r} 2=.30)$ a partir de 3 predictores significativos: expectativas de compromiso, apoyo social percibido y puntaje de PSU.

Las NEM, el dominio de herramientas informáticas y la calidad de las herramientas informáticas no fueron predictores significativos de la expectativa de desempeño durante el semestre (Tabla 1).

El testeo de un modelo de regresión lineal múltiple para predecir la intención de abandono de la universidad mostró un porcentaje de la varianza explicada en esta variable del $20.9 \%(\mathrm{r} 2=.209)$ a partir de 2 predictores significativos: expectativas de desempeño y expectativas de compromiso durante el semestre.

La PSU, las NEM, el apoyo social percibido, el dominio de herramientas informáticas y la calidad percibida de las herramientas informáticas no fueron predictores significativos de la intención de abandono de la universidad (Tabla 2).

Tabla 1. Regresión lineal múltiple sobre la variable dependiente "Expectativas de desempeño durante el semestre"

\begin{tabular}{lccccc}
\hline \multirow{2}{*}{ Modelo } & \multicolumn{2}{c}{ Coef. no estandarizados } & Coef. estandarizados & \multirow{2}{*}{ T } & Sig. \\
\cline { 2 - 5 } & $\mathbf{B}$ & Error estándar & Beta & & \\
\hline (Constante) & 10.476 & 3.181 & & 3.294 & .001 \\
\hline $\begin{array}{l}\text { Expectativas de compro- } \\
\text { miso }\end{array}$ & .343 & .044 & .387 & 7.829 & .000 \\
\hline Apoyo social & .046 & .014 & .168 & 3.266 & .001 \\
\hline $\begin{array}{l}\text { Calidad de las herramientas } \\
\text { informáticas }\end{array}$ & .040 & .066 & .034 & .599 & .550 \\
\hline $\begin{array}{l}\text { Dominio de las herramien- } \\
\text { tas informáticas }\end{array}$ & .259 & .142 & .097 & 1.823 & .069 \\
\hline $\begin{array}{l}\text { Notas de enseñanza media } \\
\text { (NEM) }\end{array}$ & .424 & .431 & .046 & .984 & .326 \\
\hline Puntaje en la PSU & .007 & .003 & .129 & 2.707 & .007 \\
\hline
\end{tabular}


Tabla 2. Regresión lineal múltiple sobre la variable dependiente "Intención de abandono"

\begin{tabular}{lccccc}
\hline \multirow{2}{*}{ Modelo } & \multicolumn{2}{c}{ Coef. no estandarizados } & Coef. estandarizados & & S \\
\cline { 2 - 5 } & $\mathbf{B}$ & $\begin{array}{c}\text { Error están- } \\
\text { dar }\end{array}$ & Beta & & \\
\hline (Constante) & 20.699 & 5.653 & & 3.662 & .000 \\
\hline Expectativas de compromiso & -.220 & .083 & -.151 & -2.633 & .009 \\
\hline Expectativas de desempeño & -.455 & .097 & -.277 & -4.688 & .000 \\
\hline Apoyo social & -.048 & .025 & -.105 & -1.885 & .060 \\
\hline Calidad de las herramientas informáticas & -.025 & .116 & -.013 & -.211 & .833 \\
\hline Dominio de las herramientas informáticas & -.354 & .250 & -.081 & -1.417 & .157 \\
\hline Notas de Enseñanza Media (NEM) & -.807 & .754 & -.054 & -1.070 & .285 \\
\hline Puntaje en la PSU & -.002 & .005 & -.023 & -.458 & .647 \\
\hline
\end{tabular}

\section{Discusión y conclusiones}

El abandono universitario y los problemas vinculados con el desempeño son problemáticas en las que diferentes aspectos de la vida educativa de los alumnos se ven influidos. Estos aspectos educativos podrían verse aún más afectados debido al actual contexto de educación virtual de emergencia producto de la pandemia del COVID-19. Además, la importancia relativa de las variables que son predictores relevantes del abandono podría ser un indicio que señale diferencias con un contexto regular de enseñanza, debido a las diferencias entre una educación regular y el sistema de enseńanza virtual de emergencia adoptado. Sin embargo, para conocer cabalmente el alcance de esta afirmación es necesario realizar una investigación con muestras representativas en contextos educativos presenciales y virtuales.

Por ello, la presente investigación tuvo como objetivo evaluar la capacidad predictiva de las expectativas de compromiso, el apoyo social, la calidad y dominio de los recursos informáticos, las NEM y la PSU sobre las expectativas de desempeño y la intención de abandono durante el semestre académico afectado por el COVID-19 en estudiantes universitarios chilenos.

En primer lugar, es importante entender que el abandono universitario, lejos de ser un fenómeno producido por una única causa, constituye un producto de múltiples variables que interaccionan entre sí, entre las que se encuentran variables no solo académicas y motivacionales, sino también sociales y afectivas. En este sentido, y en condiciones normales de presencialidad regular, variables asociadas al perfil de ingreso del estudiante, como su puntaje en la PSU o en las NEM (Jorquera et al., 2018; Vergara et al., 2016), así como variables asociadas a la experiencia socioacadémica, como el compromiso académico (Maluenda, et al., 2021; Maroco et al., 2016), el apoyo social percibido por los pares (Maluenda et al., 2020) o las metas que tienen los propios estudiantes (Maluenda et al, 2019), son algunas de las variables de interés para poder predecir el abandono universitario.

En segundo lugar, el paso a una modalidad de educación online producto de la pandemia del COVID-19 ha supuesto una constante adaptación por parte tanto del alumnado como del cuerpo docente para poder hacer frente a las exigencias de este nuevo contexto. Así, perfiles de diferente tipo socioeconómico se han visto en la necesidad de enfrentarse a dichas medidas, algo que antes de la pandemia ya suponía diferentes trayectorias en el desempeño dentro de la educación superior en la sociedad chilena, debido a los altos niveles de desigualdad predominantes, como mostraron los estudios de Ayala y Atencio (2018).

Por todo ello, en la presente investigación se ha observado que, en el caso de la intención de abandono, tanto las expectativas de desempeño como el compromiso académico aparecen como predictores 
significativos, de modo similar a un contexto de estudio regular. Estos resultados concuerdan con diferentes estudios, como los de Pineda-Báez et al. (2014), en los que se observó que existían correlaciones estadísticamente significativas entre los índices del compromiso estudiantil y el promedio académico, los cuales llevan a reflexionar sobre aspectos claves para el fortalecimiento de las experiencias educativas que se ofrecen al estudiante universitario.

Estos fueron predictores fuertes que indican que tanto la expectativa sobre el nivel de interés por los estudios, el nivel de participación y el esfuerzo intelectual que se espera realizar durante el curso de los estudios, como las expectativas sobre los resultados académicos, son aspectos que pueden afectar tempranamente el análisis y la toma de decisiones de los estudiantes respecto de permanecer o abandonar la universidad.

Del conjunto de variables evaluadas, el apoyo social no ha sido observado como predictor del abandono universitario, algo que contrasta con diferentes estudios en los que se ha observado que las variables sociales tales como la adaptación social poseen una gran fuerza predictora (Castro-López et al., 2021). Esto puede deberse a que en el análisis sobre el apoyo social se tomó como medición el apoyo de los docentes, de los compañeros y de la familia de forma conjunta. Así, una posible explicación podría ser que únicamente uno de esos parámetros de medición, posiblemente el apoyo recibido por los pares, podría ser en realidad predictor del abandono universitario.

Otra posible explicación podría ser que los alumnos no hayan contado con expectativas claras sobre las relaciones de apoyo disponibles debido al breve tiempo en el que han estado expuestos al nuevo escenario académico, teniendo en cuenta el momento en el que se realizó la investigación.

Por otro lado, las NEM y la PSU no resultaron predictores estadísticamente significativos, algo que, como se exponía con anterioridad, supone una evidencia contrapuesta a investigaciones anteriores (Jorquera et al., 2018), por lo que queda abierto el debate. Una posible explicación puede ser que el valor predictivo de las NEM y la PSU pierde significatividad debido al presente escenario de incertidumbre. Es decir, el nivel de preparación previo podría tener mucha menos fuerza predictiva en la intención de abandono en escenarios que distan de la situación de educación regular, si bien en situaciones de completa normalidad sí serían predictores estadísticamente significativos. En otras palabras, en escenarios de incertidumbre, es posible que la preparación académica disminuya de importancia sobre las expectativas que se tienen.

Por otro lado, en la presente investigación se tomó como muestra estudiantes de diferentes cursos dentro de la universidad. Esto también podría afectar la capacidad predictiva, ya que las NEM y la PSU podrían ser predictoras sobre todo en los alumnos de los primeros cursos, pero no en los de años superiores. Según los estudios de Montmarquette et al. (2001), el rendimiento académico previo (traducido en las notas de acceso a la universidad) es el factor académico más relacionado con el abandono junto con el rendimiento académico en el primer curso de la universidad, lo cual ejerce un rol crucial en la relación de permanecer o no en la titulación cursada. Esto, además, pudo comprobarse en los estudios de Esteban et al. (2016), en los que se observó que las variables de tipo académico son las que tienen mayor peso a la hora de predecir el abandono universitario, y el rendimiento académico antes del ingreso en la universidad es una de las de mayor valor predictivo.

En cuanto a la expectativa de desempeño, los predictores significativos han sido las expectativas de compromiso y el apoyo social, al igual que en otras investigaciones (Díaz-Mujica et al., 2018; López-Angulo et al., 2019; Maluenda et al., 2020). Esto reafirma el valor que tiene el nivel de interés por los estudios cursados, el nivel de participación y el esfuerzo intelectual que se involucra en los estudios, además del grado de apoyo percibido en el entorno inmediato para mantener un buen desempeño en los estudios. La PSU también demostró ser un predictor significativo, lo que puede ser indicativo del peso de la autoeficacia académica sobre el pronóstico del éxito académico en un escenario educativo complejo. 
Por su parte, ni la calidad ni el dominio de herramientas informáticas fueron predictores significativos en ninguna de las variables dependientes del estudio. Esto puede deberse a que son aspectos instrumentales que pueden ser modificados de manera sencilla, ya sea por los estudiantes, sus familias o la institución educativa. Además, es importante entender que estas variables podrían haberse visto afectadas por el contexto del alumnado: desde los primeros meses de la pandemia, los alumnos habían sido informados de la posibilidad de recibir apoyo tecnológico y económico, lo que podría indicarnos que tomaron dicha información con cierta seguridad, algo que se ve reflejado en el análisis de datos realizado.

En definitiva, múltiples son las variables que pueden afectar la intención de abandonar los estudios universitarios, así como la expectativa de desempeño. Sin embargo, parece importante tener en cuenta que el contexto en el que los estudiantes realizan sus carreras es importante y puede afectar la capacidad de predicción de dichos fenómenos. Los contextos de emergencia, como el de la actual pandemia por el COVID-19, suponen cambios en la forma en la que se imparten los contenidos, así como en el formato en el que estos tienen lugar (en este caso, de forma virtual).

Por lo tanto, las futuras investigaciones deberán tener en cuenta estas peculiaridades para poder observar cuáles son las variables con mayor fuerza explicativa de dichos fenómenos considerando las diferencias observadas según el contexto.

Este último aspecto constituye un aporte sustantivo de esta investigación, puesto que: a) realza la importancia de estudiar las variables de forma contextualizada, b) aporta evidencia sobre la operación de los predictores socioacadémicos de la intención de abandono durante la pandemia del COVID-19, y c) destaca la relevancia del análisis temprano de variables modificables para predecir y tomar decisiones que sirvan al mejoramiento de las condiciones en las que se desarrolla el proceso educativo de modo que reduzcan el abandono de los estudios.

Su limitación más importante radica en el alcance de la muestra, que cubre un pequeńo porcentaje del universo y no fue seleccionada de manera aleatoria, lo que puede afectar la posibilidad de extrapolación de los resultados observados y sus conclusiones.

\section{Agradecimientos y reconocimientos}

El presente trabajo de investigación contó con el financiamiento de Becas Doctorado Nacional CONICYT Folio 21180223.

\section{Referencias bibliográficas}

Ato, M., López, J. J., \& Benavente, A. (2013). Un sistema de clasificación de los diseños de investigación en psicología. Anales de Psicología, 29(3), 1038-1059. https://doi.org/10.6018/analesps.29.3.178511

Ayala, M. C., \& Atencio, I. J. (2018). Retención en la educación universitaria en Chile. Aplicación de un modelo de ecuaciones estructurales. Revista de la Educación Superior, 47(186), 93-118.

https://doi.org/10.36857/resu.2018.186.350

Benítez-Zavala, A. (2016). Abandono escolar: modelos que lo explican y programas para atenderlo. Una revisión crítica. Revista de Educación y Desarrollo, 37, 27-32. https://r.issu.edu.do/l?l=10325Pqd

Castro-López, A., Cervero, A., Galve-González, C., Puente, J., \& Bernardo, A. B. (2021). Evaluating critical success factors in the permanence in Higher Education using multi-criteria decision-making. $\mathrm{Hi}$ gher Education Research \& Development. https://doi.org/10.1080/07294360.2021.1877631

Deaño, M., Alfonso, S., Conde, Á., García-Señorán, M., \& Tellado, F. (2014). Expectativas universitarias y predicción del rendimiento académico. Revista Electrónica de Psicología, 4(1), 127-141.

https://r.issu.edu.do/l?l=10326Hmk

Díaz-Mujica, A., García, D., López, Y., Maluenda, J., Hernández, H., \& Pérez-Villalobos, M. (14-16 de noviembre de 2018). Mediación del ajuste académico entre variables cognitivo-motivacionales y la intención de abandono en primer año de universidad. En Octava conferencia latinoamericana sobre el abandono en 
la educación superior. Ciudad de Panamá, Panamá. https://r.issu.edu.do/l?l=505u2B

Duque, J., Montes, I., Rodríguez, S., \& Jaramillo, A. (2014). Intención de abandono en estudiantes de pregrado: factores y soluciones. Center for Research in Economics and Finance (CIEF), Working Papers, (14-15), 1-69. https://r.issu.edu.do/l? $1=10335 \mathrm{HHk}$

England, C. (2012). Teoría Social Cognitiva y Teoría de Retención de Vincent Tinto: Marco Teórico para el estudio y medición de la auto-eficacia académica en estudiantes universitarios. Revista Griot, 5(1), 28-49. https://r.issu.edu.do/l?l=10327JPG

Esteban, M., Bernardo, A. B., Tuero, E., Cerezo, R., \& Núñez, J. C. (2016). El contexto sí importa: identificación de relaciones entre el abandono de titulación y variables contextuales. European Journal of Education and Psychology, 9, 79-88.

http://doi.org/10.1016/j.ejeps.2015.06.001

Jorquera, Ó., Farías, J., \& González, P. (14-16 de noviembre de 2018). ¿Cuáles son los factores asociados al abandono de los estudiantes de programas de equidad en Chile? El caso de una universidad tradicional. En Octava conferencia latinoamericana sobre el abandono en la educación superior. Ciudad de Panamá, Panamá.

https://r.issu.edu.do/l?l=551Pyd

López-Angulo, Y., Maluenda, J., \& Varas, M. (octubre de 2019). Factores personales e institucionales vinculados con el compromiso académico en estudiantes universitarios chilenos de ingeniería. En IX Congreso latinoamericano sobre abandono en educación superior. Bogotá. https://r.issu.edu.do/l?l=552GBw

Maluenda, J., Flores-Oyarzo, G., Bernardo, A., \& Díaz, A. (2021). Correlatos conductuales del Compromiso Académico en estudiantes de ingeniería chilenos. Trilogía Ciencia Tecnología Sociedad, 13(24), e-1754.

https://doi.org/10.22430/21457778.1754

Maluenda, J., Flores-Oyarzo, G., \& Varas, M. (2020). Comportamientos interpersonales del docente asociados al compromiso académico de estudiantes de primer año de Ingeniería. Revista de Estudios y Experiencias en Educación, 19(39), 145-161.

https://doi.org/10.21703/rexe.20201939maluenda8

Maluenda, J., López, Y., Varas, M., Bernardo, A., Díaz, A., \& Moraga, F. (2019). Predictores psicosociales de la intención de abandono en estudiantes de ingeniería chilenos. En IX Congreso Latinoamericano sobre Abandono en Educación Superior. Bogotá. https://r.issu.edu.do/l?l=5534YY

Maluenda, J., Varas, M., Díaz, A., \& Bernardo, A. (2020). Propiedades psicométricas del University Student Engagement Inventory en estudiantes de ingeniería chilenos. Revista Iberoamericana de Diagnóstico y Evaluación - e Avaliação Psicológica RIDEP, 57(4), 77-90.

https://doi.org/10.21865/RIDEP57.4.06

Maroco, J., Maroco, A. L., Campos, J. A. D. B., \& Fredricks, J. A. (2016). University student's engagement: development of the University Student Engagement Inventory (USEI). Psicologia: Reflexão e Crítica, 29(1), 21.

https://doi.org/10.1186/s41155-016-0042-8

Moneta, A. M. (2019). Determinantes del desempeño académico en educación a distancia: aplicación de un modelo de ecuaciones estructurales [Tesis de Maestría, Universidad Nacional de Córdoba, Argentina]. https://r.issu.edu.do/l?l=10328DBB

Montmarquette, C., Mahseredjian, S., \& Houle, R. (2001). The determinants of university dropouts: A bivariate probability model with sample selection. Economics of Education Review, 20, 475-484.

https://r.issu.edu.do/l?l=10329oWl

Orozco, H., Suarez, J., Olarte, M., Cabanzo, C., \& Beltrán, A. (2017). Estudio factores asociados a la deserción estudiantil en la Universidad Minuto De Dios de la sede virtual y a distancia. En Séptima conferencia latinoamericana sobre el abandono en la educación superior. Universidad Nacional de Córdoba, Argentina. https://r.issu.edu.do/l?l=10330cvr

Ortega-Medellín, M. P., Marín-Martínez, V. M., Reynoso-Orozco, O., \& Lara-García, B. (2020). 
Predictores del desempeño académico del alumnado del Centro Universitario de Ciencias de la Salud de la Universidad de Guadalajara. Revista de Educación y Desarrollo, 54, 31-41.

https://r.issu.edu.do/l?l=10331udx

Pineda-Báez, C., Bermúdez-Aponte, J. J., Rubiano-Bello, Á., Pava-García, N., Suárez-García, R., \& Cruz-Becerra, F. (2014). Compromiso estudiantil y desempeño académico en el contexto universitario colombiano. Relieve, 20(2).

https://doi.org/10.7203/relieve.20.2.4238

Pleitz, J. D., MacDougall, A. E., Terry, R. A., Buckley, M. R., \& Campbell, N. J. (2015). Discrepancy between expectations and experiences on College student retention. Journal of College Student Retention: Research, Theory \& Practice, 17(1), 88-104. https://doi.org/10.1177/1521025115571252

Saldaña, M., \& Barriga, O. (2010). Adaptación del modelo de deserción universitaria de Tinto a la Universidad Católica de la Santísima Concepción, Chile. Revista de Ciencias Sociales, XVI(4), 616-628. https://r.issu.edu.do/l?l=10332zEd
Schmitt, R. E., \& Santos, B. S. (2016). Permanencia estudiantil en la educación superior: un estudio meta-teórico basado en la bioecología del desarrollo humano. En Sexta conferencia latinoamericana sobre el abandono en la educación superior. Quito, Ecuador. https://r.issu.edu.do/l?l=10333InG

Tuero, E., Cervero, A., Esteban, M., \& Bernardo, A. (2018). ¿Por qué abandonan los alumnos universitarios? Variables de influencia en el planteamiento y consolidación del abandono. Educación XXI, 21(2), 131-154. https://doi.org/10.5944/educXX1.20066.

UNESCO (Ed). (2020). COVID-19 y educación superior: De los efectos inmediatos al dia después. Análisis de impactos, respuestas politicas y recomendaciones. Instituto Internacional para la Educación Superior en América Latina y el Caribe.

https://r.issu.edu.do/l?1=103341g5

Vergara, J., Del Valle, M., Cobo, R., Pérez, M., \& Díaz, A. (2016). Factores explicativos del abandono académico en estudiantes de pedagogía. En Sexta conferencia latinoamericana sobre abandono en educación superior. Quito, Ecuador. https://r.issu.edu.do/l?1=554jvf

\section{CÓMO CITAR:}

Maluenda-Albornoz, J., Bernardo, A. B., Galve-González, C., Flores-Oyarzo, G., Infante-Villagrán, V., \& Díaz-Mujica, A., (2021). Variables predictoras de la expectativa de desempeño y la intención de abandono en contexto de educación virtual de emergencia en estudiantes universitarios chilenos. RECIE. Revista Caribeña de Investigación Educativa, 5(2), 81-91. https://doi.org/10.32541/recie.2021.v5i2.pp81-91 